\title{
Le remdésivir chez les patients atteints de la COVID-19
}

\author{
Peter E. Wu MD MSc, Andrew M. Morris MD MSc
}

Citation : CMAJ 2021 January 25;193:E125. doi : 10.1503/cmaj.202505-f; diffusion hâtive le 6 janvier 2021

Voir la version anglaise de l'article ici : www.cmaj.ca/lookup/doi/10.1503/cmaj.202505

\section{Le remdésivir est un antiviral agissant contre une multitude de virus à ARN}

Le remdésivir est un médicament administré par voie intraveineuse qui inactive l'ARN polymérase ARN-dépendante des virus et qui agit in vivo et in vitro contre le coronavirus du syndrome respiratoire du Moyen-Orient, le coronavirus du syndrome respiratoire aigu sévère 1 (SRAS-CoV-1) et le SRAS-CoV-2 ${ }^{1-3}$. Son utilisation est autorisée au Canada, et il est distribué par Santé Canada pour les patients ( $\geq 12$ ans et $\geq 40 \mathrm{~kg}$ ) atteints de la maladie à coronavirus 2019 (COVID-19) sous oxygénothérapie ${ }^{3}$.

2

\section{Le remdésivir a été testé à titre de traitement de la COVID-19}

dans le cadre de 2 importants essais cliniques

Quatre essais randomisés contrôlés (ERC) publiés ont évalué l'utilisation du remdésivir dans le traitement de la COVID-191,2,4,5. Les 2 plus importants essais étaient l'Adaptive Covid-19 Treatment Trial (ACTT-1), un ERC avec témoins sous placebo portant sur 1062 patients $^{1}$, et l'essai Solidarity, un ERC ouvert comparant le traitement au remdésivir à la norme thérapeutique chez 6838 patients ${ }^{5}$.

\section{3}

\section{Le remdésivir pourrait réduire le temps de rétablissement,}

\section{mais pas la mortalité}

L'essai ACTT-1 a conclu que le temps médian de rétablissement clinique passait de 15 jours à 10 jours avec l'administration de remdésivir, et les plus importantes améliorations ont été observées chez les patients sous oxygénothérapie à bas débit. L'essai n'a révélé aucune différence dans la mortalité (rapport de risque 0,73; intervalle de confiance [IC] à $95 \%$ 0,52$1,03)$, mais il n'avait pas la puissance statistique nécessaire pour le faire ${ }^{1}$. L'essai Solidarity n'a pas montré une amélioration de la mortalité (taux d'incidence 0,95 ; IC à $95 \% 0,81-1,11$ ) et n'a pas non plus montré d'amélioration des résultats secondaires préétablis, soit le nombre de jours sous ventilation et la durée de l'hospitalisation ${ }^{5}$.

4 Le remdésivir n'aide pas les patients gravement malades

Des analyses par sous-groupes des essais ACTT-1 et Solidarity montrent que le remdésivir n'apporte aucuns bénéfices aux patients intubés ou sous oxygénation par membrane extracorporelle ${ }^{1,5}$. Bien que ce ne soit pas aussi sûr, il ne semblait pas non plus y avoir de bénéfices significatifs pour les patients sous oxygénothérapie à haut débit ${ }^{1,5}$.

\begin{abstract}
5 Les données sur les événements indésirables sont limitées, mais comprennent des réactions d'hépatotoxicité et d'hypersensibilité Les patients ayant d'importants taux d'enzymes hépatiques ou un débit de filtration glomérulaire de moins de $30 \mathrm{~mL} /$ min ont été exclus des essais publiés ${ }^{1,2,4,5}$. Des lésions du foie causées par le médicament ont été signalées ${ }^{6}$, et des réactions anaphylactiques ou liées à la perfusion peuvent se produire ${ }^{1}$.
\end{abstract}

\section{Références}

1. Beigel JH, Tomashek KM, Dodd LE, et al.; ACTT-1 Study Group Members. Remdesivir for the treatment of COVID-19: final report. N Engl J Med 2020;383:1813-26.

2. Wang Y, Zhang D, Du G, et al. Remdesivir in adults with severe COVID-19: a randomised, double-blind, placebocontrolled, multicentre trial. Lancet 2020;395:1569-78.

3. Update on remdesivir: continued monitoring. Ottawa: Health Canada; modified 2020 Nov. 26. Accessible ici : www.canada.ca/en/health-canada/services/drugs-health -products/covid19-industry/drugs-vaccines-treatments/ remdesivir-update.html (consulté le 8 déc. 2020).

4. Spinner CD, Gottlieb RL, Criner GJ, et al.; GS-US-540-5774 Investigators. Effect of remdesivir vs standard care on clinical status at 11 days in patients with moderate COVID-19: a randomized clinical trial. JAMA 2020;324: 1048-57.

5. WHO Solidarity Trial Consortium; Pan H, Peto R, HenaoRestrepo A-M, et al. Repurposed antiviral drugs for COVID-19: interim WHO Solidarity Trial results. N Engl J Med 2020 Dec. 2 [cyberpublication avant impression]. doi: 10.1056/NEJMoa2023184.

6. Carothers C, Birrer K, Vo M. Acetylcysteine for the treatment of suspected remdesivir-associated acute liver failure in COVID-19: a case series. Pharmacotherapy 2020;40: 1166-71. 
Intérêts concurrents : Peter Wu et Andrew Morris sont respectivement membre et président du groupe de travail ontarien sur l'établissement de guides de pratiques cliniques sur la COVID-19, un groupe de travail provincial bénévole ayant pour but de fournir des guides consensuels fondés sur des données probantes pour le traitement de la COVID-19. Aucun autre intérêt concurrent n'a été déclaré.

Cet article a été révisé par des pairs.

Affiliations : Département de médecine (Wu, Morris), Division de pharmacologie et de toxicologie clinique (Wu) et Division d'infectiologie (Morris), Université de Toronto; Division de médecine interne générale et de gériatrie $(\mathrm{Wu})$, Réseau universitaire de santé; Division d'infectiologie (Morris), Système de santé Sinai et Réseau universitaire de santé, Toronto, Ont.

Propriété intellectuelle du contenu : Il s'agit d'un article en libre accès distribué conformément aux modalités de la licence Creative Commons Attribution (CC BY-NC-ND 4.0), qui permet l'utilisation, la diffusion et la reproduction dans tout médium à la condition que la publication originale soit adéquatement citée, que l'utilisation se fasse à des fins non commerciales (c.-à-d., recherche ou éducation) et qu'aucune modification ni adaptation n'y soit apportée. Voir : https:// creativecommons.org/licenses/by-nc-nd/4.0/deed.fr.

Correspondance : PeterWu, peter.wu@uhn.ca 